\title{
A case of Brown-Sequard syndrome with associated Horner's syndrome after blunt injury to the cervical spine
}

\author{
A Edwards, R Andrews
}

\begin{abstract}
A 26 year old motorcyclist was received by the trauma team in our accident and emergency department after a head on collision with a motor vehicle. He had been correctly immobilised and his primary survey was essentially normal. He was alert and orientated with a Glasgow Coma score of 15 and had no symptoms or signs of spinal injury. His cervical spine radiography was also normal. Neurological examination however, revealed anisocoria, his left pupil being smaller than his right, and a Brown-Sequard syndrome, with a sensory level at C6. Immobilisation was maintained and he was transferred to the regional neurosurgical centre where magnetic resonance imaging revealed a contusion of the left half of the spinal cord adjacent to the 6 th cervical vertebrae. Computed tomography revealed no bony injury but spinal column instability was demonstrated after flexion-extension spinal views and he underwent surgery to fuse his spine at the C5-C6 level. This report highlights the necessity to observe strict ATLS guidelines. This must include a thorough examination of the central and peripheral nervous system where spinal injury is suspected, even in the absence of radiographic abnormality and neck pain. This article also presents the unusual phenomena of Brown-Sequard syndrome and unilateral Horner's syndrome after blunt traumatic injury to the cervical spine.

(Emerg Med f 2001;18:512-513)
\end{abstract}

Keywords: cervical trauma; Brown-Sequard syndrome

\section{Case report}

A 26 year old motorcyclist was thrown from his motorbike over the roof of a motorcar after a head on collision. He landed on his back, which was afforded some degree of protection by a Kevlar back support within his protective clothing. $\mathrm{He}$ was conscious throughout and was brought into the accident centre immobilised on a long spinal board with head blocks and a hard collar in place, secured to the board with straps and adhesive Velcro. Primary survey revealed a well maintained airway and no sign of compromised ventilation. He was haemodynamically stable with no clinical signs of chest, pelvic or abdominal injury. Initial neurological examination found him alert and orientated with a Glasgow Coma Score of 15 . His pupils were unequal, the right being two millimetres larger than the left, but direct and consensual reflexes were intact. He had minimal drooping of the right upper eye lid.

His cervical collar was removed with manual in line stabilisation and he was log rolled. Examination of his neck and back revealed no tenderness, haematoma, or deformity along the full length of his spine and a rectal examination demonstrated intact sensation and voluntary muscle contraction but reduced anal tone. Subsequent radiography of the cervical spine revealed no evidence of bony injury.

Full neurological examination demonstrated reduced motor power on the left side. Shoulder abduction and elbow flexion was diminished but movement was absent or reduced to a flicker for elbow extension, wrist flexion and extension, finger extension and flexion, and all muscle groups in the lower limb. Right sided motor function was normal. Sensation was abnormal below C6 with absent pinprick sensation on the right side and abnormal light touch and proprioception sensation on the left side. Reflexes were all reduced on the left side. Although miosis and ptosis of the right eye were present, anhydrosis was not noted.

Brown-Sequard lesion was diagnosed with the lesion present on the left side of the cord at the level of C6 in association with probable ipsilateral Horner's syndrome. In accordance with neurosurgical advice he was given high dose methyl-prednisolone. He was transferred to the regional neurosurgical centre where a computed tomography scan confirmed the absence of a bony injury. Magnetic resonance imaging revealed a spinal cord contusion in the left side of the cord at the C6 level (fig 1).

Subsequently flexion-extension views of his cervical spine were performed that showed instability at C5-C6 level and he underwent a fusion procedure. His neurological signs showed improvement over the following days and it is expected with continued rehabilitation in a dedicated centre he will continue to make progress.

\section{Discussion}

In writing this case report we aim to highlight the need to adhere to ATLS guidelines in the initial assessment of trauma patients emphasising the necessity to include an examination of the peripheral as well as the central nervous systems. Also we report the rare occurrence of a blunt injury to the cervical spine causing a Brown-Sequard syndrome with associated Horner's syndrome, without localising symptoms or signs in the neck.

The classic signs of a Brown-Sequard syndrome are well documented in the literature. 


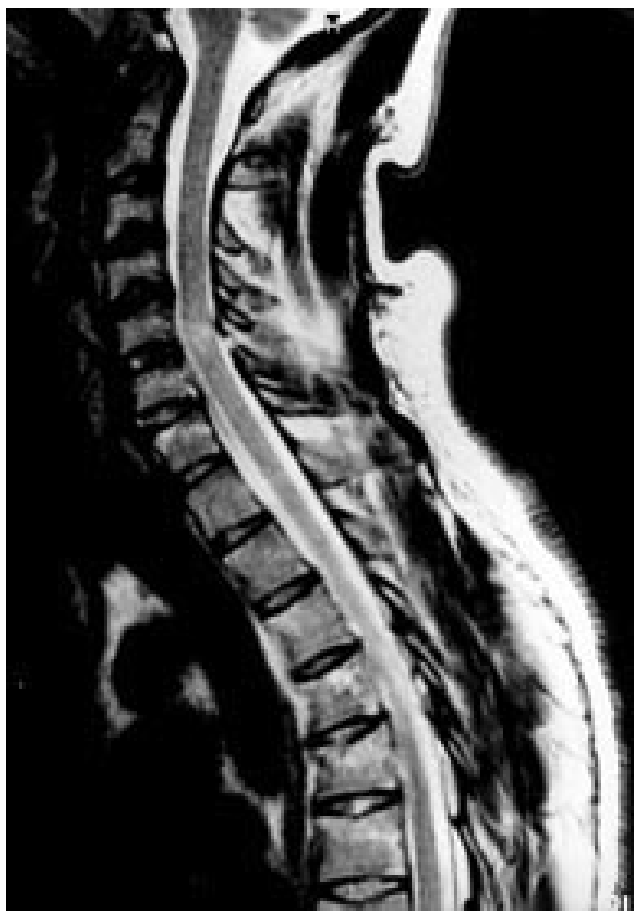

Figure 1 Sagittal section MRI of cervical spine showing a lesion at the spinal cord level of $C 6$.

Ipsilateral motor and proprioceptive loss and contralateral loss of pain and temperature sensation, or hypalgesia is present. It is a rare occurrence from any cause, but most often is associated with penetrating trauma to the spine. Associations have been made with, among others, epidural haematoma, ${ }^{12}$ cervical disc herniation, ${ }^{3}$ space occupying lesions, ${ }^{4}$ and blunt trauma. ${ }^{5}{ }^{6} \mathrm{Shen}^{2}$ reported the simultaneous presence of a Horner's syndrome caused by an epidural haematoma in a patient with Brown-Sequard syndrome from cervical trauma. Simple anisocoria may be found in up to $20 \%$ of the general population and may vary from day to day in the same person. In most patients, the degree of anisocoria will be less than $1 \mathrm{~mm}$ and there will be no ptosis, dilatation lag or vasomotor dysfunction. Other causes include local ophthalmological conditions, CN III dysfunction attributable to, for example, uncal herniation, posterior communicating artery aneurysm, tumours, strokes, diabetes, Lyme disease or alcoholism, and pharmacological glaucoma medications. Our patient had cervical trauma causing a Horner's syndrome as the aetiological factor for anisocoria. The presence of anisocoria should therefore sound alarm bells in the clinician's mind when dealing with a trauma patient. ${ }^{7}$

Recovery may be significantly better than that from penetrating trauma ${ }^{8}$ if the diagnosis is recognised early and no further injury is sustained. Immobilisation of the cervical spine and administration of high dose corticosteroids at the earliest moment should be the aim of the accepting accident department. ${ }^{9}$ Prompt referral to a neurosurgical or spinal unit is warranted for further investigation and treatment to maximise the outcome. In the trauma receiving room, principles of resuscitation must be adhered to in all cases, in particular when the diagnosis of a patient's condition is not immediately obvious. Immobilisation of the C-spine must be maintained until an injury can be ruled out clinically. Examination of the peripheral nervous system must not be overlooked in the secondary survey. Radiographs of the $\mathrm{C}$-spine alone must not lead the clinician into a false sense of security, and clinical findings both in the neck and in the neurological examination must be taken into consideration. In our case report we comment that the C-spine radiographs were normal, and examination for tenderness, a step, or swelling was negative. However, on the clinical findings in the neurological assessment we continued to assume a spinal injury.

On further imaging at a neurosurgical centre an unstable segment was demonstrated on controlled flexion-extension radiographs. MRI scans were also taken, showing cord contusion but no clear epidural haematoma despite the presence of a Horner's syndrome. Early neurosurgical intervention of decompression and stabilisation of the cervical spine may give this patient the best chance for recovery.

Doctors participating in trauma management need to be thorough in their examination of the nervous system even in the absence of neck pain and a "normal" C-spine radiograph. We recommend treatment similar to that presented in this case report for patients presenting to accident departments with cervical trauma causing a Brown-Sequard syndrome.

The authors thank Mr H Marsh for his surgical management of the patient. Mr Edwards is guarantor of the paper.

Funding: none.

Conflicts of interst: none.

1 Crabbe DC, Mendelow AD, Pharoh P, et al. Cervical spinal extradural haematoma causing a transient Brown-Sequard syndrome. F Neurol Neurosurg Psychiatry 1992;55:239.

2 Shen CC, Wang YC, Yang DY, et al. Brown-Sequard Shen CC, Wang YC, Yang DY, et al. Brown-Sequard
syndrome associated with Horner's syndrome in cervical syndrome associated with Horner's syndro

3 Rumana CS, Baskin DS. Brown-Sequard syndrome produced by cervical disc herniation: case report and literature review. Surg Neurol 1996;45:359-61.

4 Gray R. Anisocoria. In eMedicine, www.emedicine.com, Feb 2000.

5 Breuer AC, Kneisley LW, Fischer EG. Treatable extramedullary cord compression. Meningioma as a cause of the Brown-Sequard syndrome. Spine 1980;5:19-22.

6 Henderson SO, Hoffner RJ, Brown-Sequard syndrome due to isolated blunt trauma. F Emerg Med 1998;16:847-50.

7 Oller DW, Boone S. Blunt cervical spine Brown-Sequard injury. A report of three cases. Am Surg 1991;57:361-5.

8 Roth EJ, Park T, Pang T, et al. Traumatic cervical Brown-Sequard and Brown-Sequard-plus syndromes: the spectrum of presentations and outcomes. Paraplegia 1991; 29: $582-9$.

9 Delamarter RB, Coyle J. Acute management of spinal cord injury. 7 Am Acad Orthop Surg 1999;7:166-75. 